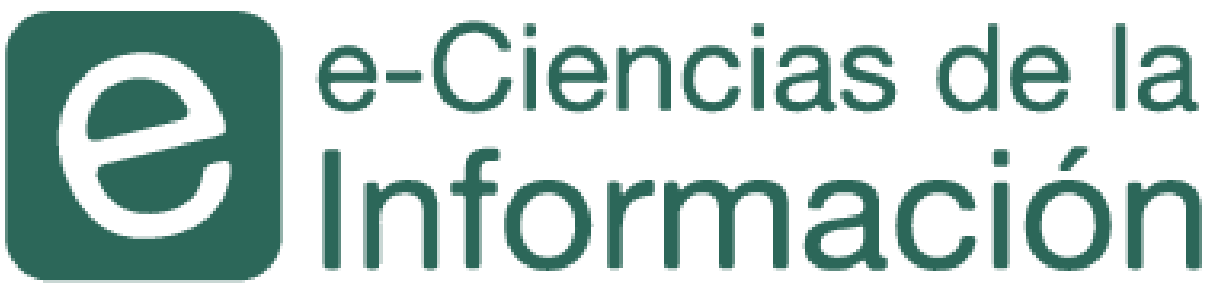

\title{
Estrategias para el fomento a la lectura: ideas y recomendaciones para la ejecución de talleres de animación lectora
}

Lorena Chaves Salgado Publicado 01 de julio, 2015 / Informe técnico 2

\section{다이(2)}

Universidad de Costa Rica

Visite el sitio web de e-Ciencias de la Información 
Estrategias para el fomento a la lectura: ideas y recomendaciones para la ejecución de talleres de animación lectora

Strategies for promoting reading: ideas and recommendations for implementing reading encouragement workshops

$\overline{\text { Lorena Chaves Salgado }}^{1}$

\section{RESUMEN}

Este trabajo plantea una guía con recomendaciones para la ejecución de talleres de animación a la lectura como un medio para fomentar el desarrollo del hábito lector y el disfrute de la lectura en la vida de los niños v las niñas.

\section{ABSTRACT}

This document presents a guide with recommendations for implementing reading encouragement workshops as a means to promote the development of reading habits and the enjoyment of reading in the lives of children.

\section{Palabras clave}

Niños, hábito de lectura, libros, lectura, técnicas, animación a la lectura, actividades

\section{Keywords}

Children, reading habits, books, reading, techniques, promoting reading, activities

Recibido: 21 de octubre 2014 | Corregido: 04 de mayo 2015 | Aprobado: 08 de junio 2015

1 Universidad de Costa Rica. Escuela de Bibliotecología y Ciencias de la Información. COSTA RICA. lorena.chaves@ucr.ac.cr 
La lectura nace como curiosidad y termina y continúa para toda la vida como algo que no solo ocupa buena parte de nuestro tiempo, sino que determina nuestro temperamento y nuestra manera de amar. El libro ha sido el acompañante más fiel y el más lúcido en todas las grandes gestas de la humanidad. La diversión que nos proporciona la lectura es la prueba más fehaciente de libertad.

Francois Jacob

\section{Introducción}

El propósito de este artículo es presentar una guía con recomendaciones para fomentar el desarrollo del hábito lector. El trabajo destaca la importancia que tiene el disfrute de la lectura en la vida de las personas, en especial cuando se es niño o niña, y la importancia de los talleres de animación a la lectura como una estrategia o recurso que busca acercar al lector/a a la construcción de un camino ameno hacia la lectura.

Según Puente Ferreras: "La lectura es una 'gozada', además de una 'necesidad'. El goce que produce el aprendizaje lector, es sobre todo atribuido al placer que causa el descubrimiento" (citado por Cerillo, 1996, p. 23)

El acontecimiento de la lectura es una experiencia que el lector debe probar por si mismo, los niños y las niñas deben aprender antes que nada a desarrollar su afición por la literatura experimentando de forma personal el disfrute que esta les brinda, leer debe ser un acto entretenido que permita desarrollar la imaginación en el tiempo y en el espacio. La lectura debe ser protagonista en la vida del niño/a desde su más tierna infancia; aun cuando todavía no haya aprendido a leer, el solo mirar imágenes en un libro le ayudará para interpretar la realidad, ya que estas cumplen la función de operar al mismo nivel del texto escrito a la hora de captar el sentido de este. La lectura le abre al niño/a el conocimiento del mundo, de los objetos con sus colores y sus formas; el niño o la niña que se verá rodeado de mensajes lingüísticos los irá descodificando poco a poco, a medida que se adentre en la lectura del texto.

Aprender a leer constituye un lento caminar hasta llegar a descubrir el sentido mágico que transmiten las palabras como portadoras de significados a través de los libros. El acto de leer permite al niño/a construir y generar ideas nuevas, ser más crítico/a y creativo/a, y expresar sus pensamientos y emociones de una forma más clara con la fuerza que le proporcionaran las palabras en una estrecha relación con el mundo exterior.

La lectura implica diversas facetas y un encuentro progresivo con el libro; por lo tanto, constituye un proceso de aprendizaje que el/la niño/a debe ir asimilando a su propio ritmo. Aunque un/a niño/a este alfabetizado/a esto no significa que vaya a ser un lector/a, lo será hasta que adquiera el hábito de leer, porque no se llega a ser lector/a de manera automática. Todo lo contrario, esta actividad personal es construida y desarrollada por la propia persona lectora a través del acercamiento y disfrute según acceda a la diversidad de obras literarias existentes.

En este proceso, es de suma importancia que el niño o niña reciba la motivación necesaria para que su encuentro con los libros se realice de una manera amena, considerando sus gustos y sus preferencias por los textos que desee leer. Hablamos entonces de un encuentro en libertad donde 
se multipliquen sus afectos y emociones hacia la actividad lectora, que viene a representar un abanico de posibilidades donde el libro debe ocupar un sitio privilegiado en la vida del niño/a.

Es necesario potenciar en él/ella su capacidad lectora y hacer que las personas no lectoras o lectoras pasivas se interesen por poner en práctica la lectura activa, proceso que debe alimentarse desde tres sitios clave como la escuela, la biblioteca (tanto pública como escolar) y el hogar. En el seno familiar es preponderante el papel de las primeras lecturas que el/la niño/a realice, ya que de una u otra forma aportaran elementos importantes de la experiencia formativa que le darán a este/a las claves del camino hacia su formación lectora:

El niño recibe en sus primeros años de vida la impronta de su entorno familiar, de modo que aquellos sujetos que crecen en un entorno lector, resultan más proclives a contemplar la presencia del libro en su vida cotidiana, frente a aquellos que lo han conocido. Al respecto conviene sensibilizar a los padres y madres, en aplicación a su paternidad o maternidad responsable, la responsabilidad que les confiere ésta, en búsqueda constante de un enriquecimiento cultural, principalmente a través de la lectura constructiva y enriquecedora. (Quintanal, 2005, p. 21)

Un ambiente que brinde las condiciones idóneas al niño/a le abrirá las puertas a una experiencia enriquecedora y sorprendente que lo acerque a los libros para descubrir que la aventura de leer es una revelación y una curiosidad por conocer mundos distintos. En el desarrollo del proceso de motivar al niño/a a leer, destaca la importancia de acercarlo/a a la lectura activa, la cual le permitirá adentrarse al encuentro con los libros de forma participativa, que da paso a una experiencia lúdica, enriquecedora y emocionante.

En este proceso de construcción del acercamiento a la lectura, la animación lectora constituye un medio que abarca estrategias y actividades que como fin primordial impulsan y atraen a las personas no solo a leer por leer un libro, sino todo lo contrario, despiertan en la persona usuaria -en este caso en los niños y las niñas- una mejora en el hábito lector para que este vaya en aumento convirtiendo la lectura en una actividad mediada por el gozo donde no tenga cabida la imposición de leer un texto.

Dentro de las actividades de animación a la lectura que ayudarán al niño o niña a mejorar esta práctica, los talleres constituyen una estrategia muy valiosa y dinamizadora, ya que permiten llevar a cabo multiplicidad de actividades para motivar a las personas usuarias, potenciales lectoras, a participar de espacios de lectura recreativa. Los talleres de lectura pueden estar estructurados de muy diversas maneras; esto dependerá de la creatividad del animador que esté a cargo de realizar la estrategia, si se considera muy importante que estas actividades sean de carácter lúdico y que no sean impuestas. De lo contrario, se corre el riesgo que, al haber imposición, la persona infante se sienta presionada y desista de leer: la magia del acto de leer debe ser llevada a cabo de una manera voluntaria, con un sentimiento de fascinación y de gozo como nos dice Michael Ende en este bello pasaje de su novela La historia interminable:

La pasión de Bastián Baltasar Bux eran los libros. Quien no haya pasado tardes enteras delante de un libro, con las orejas ardiéndole y el pelo caído por la cara, leyendo y leyendo, olvidado del mundo y sin darse cuenta que tenía hambre o se estaba quedando helado... Quien nunca haya leído en secreto a la luz de una linterna, bajo la manta porque Papá o 
Mamá o alguna otra persona solícita le ha apagado la luz con el argumento bien intencionado de que tiene que dormir, porque mañana hay que levantarse temprano.... Quien nunca haya llorado abierta o disimuladamente lágrimas amargas, porque una historia maravillosa acaba y había que decir adiós a personajes con los que había corrido tantas aventuras, a los que quería y admiraba, por los que había temido y rezado, y sin cuya compañía la vida le parecería vacía y sin sentido... Quien no conozca todo esto por propia experiencia no podrá comprender probablemente lo que Bastián hizo entonces. (Ende, 1993, p. 12)

Lograr que la niña o niño descubra las potencialidades que contienen los libros es posible por medio de una animación lectora fundamentada en buenas propuestas bien organizadas y en actividades reales que respondan a las demandas de los niños y las niñas en un ambiente creativo, entretenido y agradable, que representen un camino directo hacia la lectura. Uno de los objetivos principales de la animación es plantear una forma diferente de leer, que permita al niño/a descubrir el entorno que lo/a rodea, de manera que este/a pueda experimentar el acto de leer como su centro de interés de forma voluntaria y comprenda lo que lee, lo disfrute de la mejor manera y además medite obteniendo una enseñanza para su crecimiento personal.

La animación a la lectura, nos dice Carmen Domech, es "una actividad que se propone el acercamiento y la profundización en los libros de una manera creativa, lúdica y placentera" (Domech, 2004, p.20). Es importante tener en cuenta que llegar a ser lector/a es un proceso que responde a un tiempo distinto para cada persona, que irá creando sus propios esquemas y su forma de leer hasta llegar a alcanzar esta práctica. Por lo tanto, desde muy temprana edad se hace indispensable inculcar el hábito de la lectura como una fuente inagotable de satisfacción, como un compromiso personal de cada lector y una reflexión propia. Lo cual hace que se tome conciencia del entorno que nos rodea, como una forma de analizar y de ver el mundo desde nuestras propias perspectivas.

\section{Talleres de estrategias de Animación Lectora}

Se presenta a continuación un planeamiento de talleres para trabajar tanto desde el aula como desde la biblioteca pública o escolar. El objetivo de lo anterior es impulsar al niño o niña a leer y a desarrollar sus pensamientos y experiencias de vida de una manera integral. Cabe destacar que toda programación de animación debe ser planeada con una metodología de trabajo que permita flexibilizar dicha práctica para así poder responder a las necesidades de las personas participantes. No obstante, a la vez, lo anterior exige una planificación donde la persona animadora de los talleres trabaje de forma activa y responsable para así potenciar la literatura de forma amena y lúdica.

Este planeamiento comprende 8 sesiones para llevar a cabo con niños y niñas con edades entre los 8 y los 12 años. Para estructurar las sesiones, se tomó como referencia el libro de Animación a la Lectura: con nuevas estrategias de la autora española Montserrat Sarto (2010); se hizo una adaptación de la metodología utilizada por ella para desarrollar talleres de lectura. La periodicidad de las animaciones es de dos sesiones por mes, para darles a los niños tiempo de que puedan leer, ya que se va a trabajar con libros completos, no solo con parte de estos. Además, se plantea esta periodicidad porque algunas de las sesiones tienen también reuniones cortas para dar indicaciones 
previas de ciertas estrategias que se seguirán. Se abarcará el género de novela corta, cuento y poesía.

\subsection{Primer taller}

Actividad para niños de 8 a 9 años con el libro Paulina y el caracol, de Floria Jiménez (2000). La autora lo escribió para Paulina, su nieta; en él habla de una niña que despertó caracoles que estaban dormidos en el fondo de su imaginación. El tema central de los poemas es la posibilidad que se tiene de soñar por medio de la esperanza.

\section{a) Título del taller:}

Soy un poeta

Se intenta aquí que los niños y las niñas se familiaricen con el texto poético.

\section{b) Dirigido a:}

Un grupo de 10 niños y niñas, a quienes les debe gustar la expresión oral, en este caso recitar.

\section{c) Objetivos:}

-Entender los poemas

-Lograr construir nuevas composiciones poéticas

-Despertar la imaginación

-Desarrollar la escucha, pronunciación y dicción

d) Persona responsable de la ejecución del taller:

La persona animadora ${ }^{2}$ en esta actividad debe tener facilidad para la lectura en voz alta. Tener, además, buena entonación y buena dicción.

e) Materiales que se van a requerir:

Un grupo de 10 niños y niñas, a quienes les debe gustar la expresión oral, en este caso recitar.

f) Descripción de la puesta en práctica de la actividad:

Los niños y niñas deben leer con anticipación los poemas del libro; luego, se les preguntará cuáles les han gustado más y se comentarán. Lo anterior se realizará para propiciar una conversación y escuchar las opiniones y gustos de todas las personas participantes. Luego, el animador debe entregar en una ficha a cada participante con alguno de los poemas; después de la lectura de cada uno de ellos, se recogerán las fichas. De inmediato, se les entregará a los niños y niñas los mismos poemas elegidos, pero cada verso escrito en una ficha diferente (a modo de rompecabezas). Las personas participantes deben unir los versos para lograr una nueva composición; deben leer en voz alta sus creaciones para compartirlas con el grupo.

\section{g) Tiempo necesario para llevar a cabo el taller:}

La actividad puede durar una hora y cuarto, que será suficiente, ya que los poemas son cortos; también se podrá hacer lectura y discusión. 
h) Limitaciones que puede presentar la estrategia:

Una limitación puede ser que los niños y las niñas tengan poca familiaridad con la poesía y no sientan mucho interés en leerla. Dependerá del animador o animadora motivar con anticipación al niño/a respecto a este género para que el sienta interés y curiosidad de leer algo diferente.

\section{i) Recomendaciones al animador o animadora:}

Es conveniente que el animador o animadora, al igual que está motivando a los niños y niñas a que lean poesía, lo haga él misma o ella misma también, para que experimente leer textos poéticos y vaya conociendo como abordarlos en la animación.

\subsection{Segundo taller}

Actividad para niños y niñas de 8 años con el cuento "Aladino y la lámpara maravillosa". Este texto relata la conocida historia de Aladino del libro de "Las mil y una noches".

a) Título del taller:

¿Quién es el personaje?

En esta estrategia los niños y las niñas van a incursionar en la lectura de un cuento corto clásico de la literatura infantil.

\section{b) Dirigido a:}

Un grupo de 10 niños y niñas, que deben tener buena memoria para poder recordar características de los personajes del cuento.

\section{c) Objetivos:}

-Acercarse a la lectura de cuentos

-Ejercitar la memoria

-Desarrollar la expresión oral por medio de la descripción

d) Persona responsable de la ejecución del taller:

El animador o animadora en esta actividad debe saber cómo propiciar que los niños y las niñas se expresen oralmente.

e) Materiales que se van a requerir:

El cuento "Aladino y la lámpara maravillosa", fichas de colores, lapiceros y una caja.

\section{f) Descripción de la puesta en práctica de la actividad:}

Se pide a alguno de los niños o niñas que voluntariamente salga del salón. Se reparte a cada una de las personas participantes que se ha quedado una ficha y un lapicero. Se les pide a los niños y niñas que discutan sobre los personajes del cuento y las aventuras de los mismos que más les han llamado la atención. Después de llegar a un consenso, se escribirá en una ficha el nombre de un personaje; luego esta se depositará en una caja. Se hará entrar al niño/a que estaba afuera y cualquiera de los niños o niñas que sí estaba en el salón, de forma voluntaria, debe tomar una ficha de la caja y empezar a darle pistas, al niño o niña que acaba de entrar al aula, para que adivine de cual personaje se trata. 
g) Tiempo necesario para llevar a cabo el taller:

Esta actividad debe tener una duración de una hora y media para que los niños y niñas tengan tiempo de discutir sobre los personajes de la historia y escribir sus nombres en las fichas.

h) Limitaciones que puede presentar la estrategia:

Una limitación puede ser que haya algún niño o niña muy tímido/a que no quiera hablar delante de todo el grupo. Otra puede ser que alguno de los que toman las fichas para dar las pistas sobre los personajes no logre expresarse bien de forma oral.

i) Recomendaciones a la persona animadora:

El animador o animadora en esta actividad debe ser una persona con capacidad de impulsar a los niños y niñas a expresarse; además, debe procurar que sea una sesión muy dinámica, ya que hay comentarios e intercambio de opiniones acerca de los personajes.

\subsection{Tercer taller}

Actividad para niños y niñas de 10 años con Un rincón para los sueños de Clara Acuña (2000). Este libro contiene cuentos cortos que hablan sobre la búsqueda del bien y los valores; entre los últimos se encuentran: el amor por la naturaleza y por los demás, el respeto por las tradiciones y la importancia de mantener a la familia unida.

a) Título del taller:

Te recomiendo que leas mi cuento

En esta estrategia los niños y las niñas van a incursionar en la lectura de cuentos.

\section{b) Dirigido a:}

Un grupo de 10 niños/as; deben tener capacidad crítica para poder discutir el tema de los valores, que es el eje central de todos los cuentos.

\section{c) Objetivos:}

-Analizar el tema de los valores.

-Despertar el sentido crítico.

-Desarrollar el análisis de los contenidos.

d) Persona responsable de ejecutar el taller:

El animador o animadora en esta actividad debe tener facilidad para la discusión y sensibilidad ante temas polémicos.

e) Materiales que se van a requerir:

El libro de Un rincón para los sueños, hojas blancas y lapiceros. 
f) Descripción de la puesta en práctica de la actividad:

Algunos días previos a la sesión principal, se realiza una sesión corta, de unos 30 minutos, con las personas participantes para explicarles qué es un valor. Los niños y niñas deben elegir el cuento que les llame la atención e identificar según su propio criterio un valor que más destaque. En la sesión principal, a cada niño/a se le dará una hoja de papel; en ella debe escribirle una carta corta a un/a amigo/a contándole sobre el cuento que leyó y resaltando el tema del valor que él/ella considere más importante. La idea es que luego los niños y las niñas hablen sobre la carta que escribieron y discutan sobre ese valor que más les llamó la atención y porque es importante en sus vidas.

g) Tiempo necesario para llevar a cabo el taller:

A esta actividad se le puede destinar una hora y media, para que las personas participantes tengan tiempo de escribir su carta, y las que deseen puedan comentarla con el grupo.

h) Limitaciones que puede presentar la estrategia:

Una limitación puede ser que alguno de los niños o las niñas tenga dificultad para identificar el valor presente en el cuento. Será tarea de la persona animadora, en caso de que esto suceda, ayudarle con una explicación breve para que lo identifique.

\section{i) Recomendaciones al animador o animadora:}

Es conveniente que la persona animadora esté muy atenta a las respuestas de los niños y las niñas, respete sus opiniones y propicie la discusión en el grupo.

\subsection{Cuarto taller}

Actividad para niños y niñas de 10 a 12 años con el libro Galipán y yo, de Floria Jiménez (2002). En esta novela, la protagonista y Galipán, un muñeco de trapo, narran situaciones relacionadas con la falta de comunicación entre padres, madres e hijos/as.

a) Título del taller:

Mi diario

En esta estrategia los niños y las niñas van a incursionar en la lectura de una novela corta.

\section{b) Dirigido a:}

Un grupo de 10 niños/as que deben tener mucha inventiva y a quienes, además, les tiene que gustar escribir.

\section{c) Objetivos:}

- Acercarse a la lectura de una novela corta.

- Despertar el sentido de la creatividad.

-Desarrollar la escritura.

d) Persona responsable de la puesta en práctica de la actividad:

El animador o animadora en esta actividad debe propiciar que los niños y las niñas desarrollen la escritura. 
e) Materiales que se van a requerir:

El libro "Galipán y yo", un cuaderno y lapiceros.

f) Descripción de la puesta en práctica de la actividad:

Realizar una sesión corta, de unos 30 minutos, unos días antes de la sesión principal para darles a los niños y las niñas distintas ideas de cómo pueden armar su diario. Ellos/as deben leer la novela con la indicación de que, conforme la vayan leyendo, escriban un diario. Al igual que la niña del cuento, deben ir narrando sus vivencias que compartirán con sus demás compañeros y compañeras del grupo de lectura el día de la actividad.

\section{g) Tiempo necesario para llevar a cabo el taller:}

Esta actividad debe ser de hora y media para que los niños y las niñas que deseen hacerlo compartan en voz alta lo que escribieron y expliquen cómo elaboraron su diario.

\section{h) Limitaciones que puede presentar la estrategia:}

Una limitación puede ser que los niños y las niñas vayan a tener que recibir acompañamiento por parte del animador o animadora en la parte de la elaboración del diario. Esto implica que la persona posiblemente tenga que invertir un tiempo extra a la sesión para atender en caso de que algún niño/a tenga dudas sobre lo que está elaborando.

\section{i) Recomendaciones al animador o animadora:}

El animador o animadora de esta actividad debe ser una persona responsable que destine tiempo para leer lo que los niños y las niñas escribieron en sus diarios y enterarse detalladamente del trabajo que cada unos de ellos/as realizó.

\subsection{Quinto taller}

Actividad para niños y niñas de 11 años con el libro Abuelo de Orlando Burgos (2004). Esta novela corta relata la historia de un niño campesino que escribe sobre su vida cerca de los años sesenta.

\section{a) Título del taller:}

Raíces nuestras

En esta estrategia los niños y las niñas van a incursionar en la lectura de una novela corta.

\section{b) Dirigido a:}

Un grupo de 10 niños/as que debe tener mucha inventiva y ser muy observador para poder recordar y narrar historias sobre su comunidad.

\section{c) Objetivos:}

- Acercarse a la lectura de una novela corta.

- Despertar el sentido de la investigación.

-Hacer una entrevista. 
d) Persona responsable de la ejecución del taller:

El animador o animadora en esta actividad debe conocer la comunidad de donde son los niños y las niñas, para así poder desarrollar el tema de las vivencias locales.

e) Materiales que se van a requerir:

El libro Abuelo, hojas de papel, goma, tijeras, dos tapas gruesas, lápices de color y materiales adicionales (fotos, dibujos, etc).

f) Descripción de la puesta en práctica de la actividad:

Los niños y las niñas deben leer la novela con la indicación de que se informen sobre la historia de su comunidad; además, requieren recopilar datos relevantes, puede ser por medio de entrevistas y de fotografías. Después, con la información que han obtenido deben elaborar un álbum con la historia de su comunidad. En él cada persona debe poner lo que más desee contar; es decir, esto debe ser su iniciativa, lo que le llamó la atención del lugar en donde vive. Se pueden también hacer dibujos si así lo desea algún niño o niña.

Al igual que el niño, que es el personaje del libro y cuenta las historias del lugar en donde él vive, los niños y las niñas al hacer su álbum estarán también contando sus historias personales. Sería interesante hacer una exposición para mostrar el trabajo elaborado por estas lectoras y lectores.

\section{g) Tiempo necesario para llevar a cabo el taller:}

Esta actividad debe ser de unas dos horas para que los niños y las niñas tengan tiempo de discutir la novela y exponer su álbum.

\section{h) Limitaciones que puede presentar la estrategia:}

Una limitación puede ser que las niñas y los niños vayan a tener que destinar más tiempo de lo habitual no solo a la lectura de la novela, sino también a la búsqueda de información sobre la comunidad.

\section{i) Recomendaciones a la persona animadora:}

El animador o animadora en esta actividad debe ser una persona responsable que destine tiempo para informarse sobre la comunidad donde viven los niños y niñas. Además, es importante el manejo del tiempo si está dentro de sus posibilidades investigar y recopilar información.

\subsection{Sexto taller}

Actividad para niñas/os de 11 a 12 años, con el libro Por la tierra de las hadas de Carlos Rubio (2001), compilación de cuentos clásicos hecha por el escritor.

a) Título del taller:

El viaje del lector

En esta estrategia los niños y las niñas van a incursionar en la lectura de cuentos clásicos. 


\section{b) Dirigido a:}

Un grupo de 15 niños/as que tengan mucha imaginación para poder crear e interesar a sus compañeros/as a leer los cuentos.

\section{c) Objetivos:}

-Acercarse a la lectura de cuentos clásicos.

-Despertar el sentido de la imaginación.

-Desarrollar la habilidad de construir manualmente un puesto ${ }^{3}$.

\section{d) Persona responsable de la ejecución del taller:}

El/la animador/a en esta actividad debe tener una buena imaginación y estar atento/a para guiar a los niños y las niñas en su proceso de creación de los puestos.

e) Materiales que se van a requerir:

Lo único requerido es el libro Por la tierra de las hadas. En esta actividad el uso de materiales es libre por parte de los niños y las niñas: son ellos/as quienes deben aportar los materiales libremente según el puesto que deseen recrear.

\section{f) Descripción de la puesta en práctica de la actividad:}

Es conveniente realizar una reunión de aproximadamente una hora con los niños y las niñas para discutir qué ideas tienen sobre la construcción del puesto. Además, habrá que formar grupos de tres integrantes: cada grupo debe elegir uno de los cuentos del libro, el que más le haya gustado; en caso de que dos grupos escojan el mismo cuento, se debe tratar de llegar a un consenso para que este no se repita. Las personas participantes asistirán a la sesión ya preparadas con sus materiales de decoración que ellas mismos han seleccionado. Luego, se les asignará un espacio en el salón donde trabajarán cada uno de los 5 grupos que irá decorando su puesto libremente de acuerdo al cuento que eligieron. El animador junto con algunas personas invitadas (pueden ser los padres y familiares de los niños participantes de la sesión) pasarán por cada puesto escuchando a las personas participantes hacer una presentación sobre el cuento que escogió su grupo y motivando a las personas visitantes a que lo lean.

g) Tiempo necesario para llevar a cabo el taller:

Esta actividad debe durar aproximadamente dos horas, para que los niños y las niñas tengan tiempo de organizar su puesto de cuentos.

\section{h) Limitaciones que puede presentar la estrategia:}

Una limitación puede ser que las personas participantes vayan a requerir de más tiempo del que habitualmente se destina para las sesiones de lectura para preparase para la actividad.

\section{i) Recomendaciones al animador:}

La persona animadora debe ser bien organizada para no dejar de lado ninguno de los detalles, ya que en esta sesión -a diferencia de otras- además del grupo de niños y niñas que asiste a las sesiones de animación a la lectura, habrá invitados/as que participaran de la misma. Esto requerirá trabajo extra; por ello, también el animador o la animadora debe evaluar si está en 
capacidad de trabajar individualmente o si necesitará ayuda de otra persona para la organización de la actividad. Asimismo, es necesario escoger bien el lugar donde la misma se va a realizar, ya que este debe contar con el espacio y las condiciones necesarias para poder montar los puestos.

\subsection{Séptimo taller}

Actividad para niñas/os de 11 a 12 años con el libro Mo de Lara Ríos (1991). Esta novela cuenta la historia del pueblo indígena Cabécar, de la región Caribe de Costa Rica.

\section{a) Título del taller:}

La caja encantada

En esta estrategia los niños y niñas van a incursionar en la lectura de una novela sobre tradiciones indígenas costarricenses.

\section{b) Dirigido a:}

Un grupo de 15 niñas/os que debe tener mucha inventiva y ser muy observadores para poder recordar y narrar historias.

\section{c) Objetivos:}

- Profundizar en el argumento de la novela.

-Despertar el sentido de la curiosidad por el texto.

-Conocer sobre la historia de los indígenas costarricenses.

d) Persona responsable de la ejecución del taller:

El animador o la animadora en esta actividad debe conocer sobre la historia de los grupos indígenas costarricenses, en especial del pueblo Cabécar.

e) Materiales que se van a requerir:

El libro de $M O$, una caja de cartón grande, tarjetas de colores y lapiceros.

\section{f) Descripción de la puesta en práctica de la actividad:}

En el centro del salón se colocará una caja de cartón grande decorada con alusiones al pueblo indígena Cabécar. En ella se pondrán los nombres de los distintos personajes de la novela; el animador o la animadora va a invitar a uno/a de los/as niños/as a pasar y tomar de la caja una de las tarjetas. Según le corresponda, el/la participante debe describir a un personaje y caracterizar su vida en el pueblo Cabécar.

\section{g) Tiempo necesario para llevar a cabo el taller:}

Esta actividad debe ser de una hora y media para que los niños y las niñas puedan expresarse con soltura y sin apuro.

h) Limitaciones que puede presentar la estrategia:

Una limitación puede ser que haya algún/a niño/a muy tímido/a y le sea difícil participar de este ejercicio oral. 
i) Recomendaciones al animador:

El animador o la animadora en esta actividad debe ser una persona con una buena capacidad de motivar a los niños y las niñas a participar. Además, debe ser paciente mientras las personas participantes leen las tarjetas y se toman un tiempo corto para pensar antes de describir a su personaje y después caracterizarlo.

\subsection{Octavo taller}

Actividad para niños y niñas de 8 a 9 años, con Cuentos con alas y luz de Ani Brenes (2001). Este libro habla sobre la felicidad y el disfrute de las cosas que nos rodean.

\section{a) Título del taller:}

Adivina si puedes

En esta estrategia los niños y las niñas van a incursionar en la lectura de cuentos.

\section{b) Dirigido a:}

Un grupo de 16 niños/as que debe tener buena memoria para poder recordar bien detalles de la historia.

\section{c) Objetivos:}

- Acercarse a la lectura de los cuentos.

- Desarrollar una buena memoria.

-Ejercitar la comprensión de la lectura.

d) Persona responsable de la ejecución del taller:

El animador o la animadora de esta actividad debe recordar muy bien todas las partes de libro para tener claras las respuestas cuando los niños y las niñas respondan a las interrogantes que se harán sobre personajes o pasajes de la historia.

\section{e) Materiales que se van a requerir:}

Libro Cuentos con alas y luz, tarjetas de colores y marcadores.

f) Descripción de la puesta en práctica de la actividad:

Se hace una baraja con unas 20 tarjetas. En cada tarjeta deben ir escritas preguntas tanto sobre los personajes como sobre eventos que acontecen en las historias. Se divide el grupo en dos equipos, los niños y las niñas de cada equipo van escogiendo una tarjeta y la persona animadora les va haciendo las preguntas que correspondan. Si algún/a niño/a de uno de los equipos no sabe la respuesta, el otro equipo podrá contestar. Al final ganará el equipo que acumule más respuestas.

g) Tiempo necesario para llevar a cabo el taller:

Esta actividad debe ser de una hora y media para que los niños y las niñas integrantes de los diferentes equipos puedan participar. 
h) Limitaciones que puede presentar la estrategia:

Una limitación puede ser que las personas participantes que sean un poco tímidas no se animen en un primer momento a participar.

\section{i) Recomendaciones al animador o la animadora:}

Quien anima esta actividad debe ser una persona con buena memoria para que no olvide detalles de los cuentos a la hora de realizar las preguntas. También, debe ser muy dinámico/a para que le dé emoción a la competencia y para que ambos equipos participen activamente.

\section{A manera de conclusión}

Toda estrategia relacionada con la animación tiene que tener como propósito principal brindar al lector o lectora caminos de comprensión y de agrado por la lectura para llegar a ser una persona lectora autónoma, crítica y de pensamiento creativo. Su utilidad debe enfocarse en destacar el papel fundamental del libro y el modo en el cual este debe pasar a formar parte de la vida integral de las personas.

Es importante resaltar que la animación a la lectura no garantiza formar lectores/as de por vida; su valor recae en mostrar que el libro es un elemento valioso y que no necesariamente debe asociarse siempre a labores de índole académica, que muchas veces resultan tediosas para los niños, las niñas y las personas jóvenes. Contrario a esta idea, la lectura debe llegar a ser entendida como una experiencia placentera, que facilite encaminarse hacia el desarrollo del hábito lector enriquecido por un ambiente de lectura que, como ya fue mencionado, comienza desde el hogar y se fortalece en la escuela. Por lo tanto, todas las actividades que se realicen deben encaminar al niño/a hacia el desarrollo de una conciencia crítica en relación con las lecturas que realice, las cuales deben proporcionarle espacios de reflexión y de interiorización.

Es necesario dirigir esfuerzos a desarrollar en niños y niñas el hábito de evolucionar de la lectura pasiva a la lectura activa, entendiendo esta como una experiencia de carácter vital en el desarrollo integral de la persona. Integrar la lectura a las prácticas cotidianas lleva a constatar que esta actividad forma parte del entorno de la persona, porque le ayudará a crecer, a evolucionar y a desarrollarse tomando plena conciencia de su ser. 


\section{Referencias}

Acuña, C. (2000). Un rincón para los sueños. San José, C.R: Editorial Costa Rica.

Aladino y la lámpara maravillosa (Cuentos Infantiles 4). (2006). Barcelona: Editorial Sol 90.

Brenes, A. (2001). Cuentos con alas y luz. San José, C.R: Editorial Costa Rica.

Burgos, O. (2004). Abuelo. San José, C.R: Editorial Costa Rica.

Cerillo, P. (1996). Hábitos lectores y animación a la lectura. Castilla de la Mancha, España: Publicaciones de la Universidad de Castilla de la Mancha.

Domech, C. (2004). Animación a la lectura: ¿Cuántos cuentos cuentas tú?. Madrid: Editorial Popular.

Ende, M. (1993). La historia interminable. Barcelona: RBA Editores.

Jiménez, F. (2000). Paulina y el caracol. San José, C.R.: Editorial Costa Rica.

Jiménez, F. (2002). Galipán y yo. San José, C.R.: Editorial Costa Rica.

Quintanal, J. (2005). La animación lectora en el aula: técnicas, estrategias y recursos. Madrid: Editorial CCS.

Ríos, L. (1991). Mo. San José, C.R.: Editorial Costa Rica.

Rubio, C. (comp.). (2001). Por la tierra de las hadas. San José, C.R.: Uruk Editores.

Sarto, M. (2010). Animación a la lectura: con nuevas estrategias. Madrid: SM. 
¿Desea publicar su trabajo? Ingrese aquí

O escríbanos a la siguiente dirección: revista.ebo@()(2)

\section{1}

Origen: respuesta a una necesidad

En el año 2011, la Escuela de

Bibliotecología y Ciencias de la Información (EBCI) de la Universidad de Costa Rica (UCR) reconoció la importancia de crear nuevas y mejores alternativas para difusión de la investigación. e-Ciencias de la Información es la respuesta a un contexto actual marcado por una mayor apertura, flexibilidad, y rigurosidad en la verificación de los datos y su procesamiento.

\section{3}

Revista de la UCR

e-Ciencias de la Información es una revista científica que aborda las nuevas temáticas de desarrollo e investigación en las Ciencias de la Información, en el ámbito nacional e internacional. Así, colabora significativamente en el progreso de esta disciplina. Por sus parámetros de calidad, pertenece al grupo de las revistas más importantes de la UCR y se encuentra ampliamente indizada en los importantes catálogos.

\section{En la actualidad}

Posicionamiento internacional

La revista admite trabajos en las siguientes áreas, siempre que se relacionen directamente con las Ciencias de la Información:

Bibliotecología, Documentación, Tecnologías de la Información y la Comunicación, Investigación, Análisis Estadísticos y Bibliometría, Archivística, Sistemas de Información, Informática, Comunicación y Biblioteas Escolares.

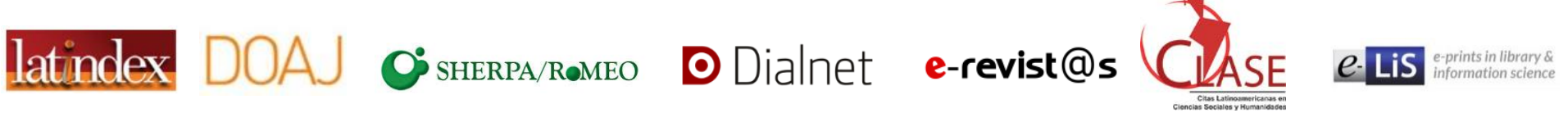

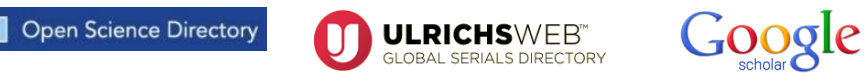

